\title{
Maintaining the stability of a leapfrog scheme in the presence of source terms
}

\author{
Cheolwan Kim* \\ W.M. Keck Foundation Lab for Computational Fluid Dynamics, Department of Aerospace Engineering, \\ The University of Michigan, Ann Arbor, MI 48109-2140, U.S.A.
}

\begin{abstract}
SUMMARY
The instability encountered by applying the upwind leapfrog method to the advection equation having a source term is resolved. The source term is eliminated by transforming the governing equation. Two types of transformation are examined and the method of the space transformation leads to a stable and accurate scheme for the one-dimensional advection equation. The method is also extended to the two-dimensional acoustic equations in polar co-ordinates. Copyright (C) 2003 John Wiley \& Sons, Ltd.
\end{abstract}

KEY WORDS: stability; transformation; leapfrog; upwind; non-dissipative

\section{INTRODUCTION}

Recently, several numerical schemes have been developed for unsteady linear problems [1-7], such as high-frequency and long-range wave propagation. These schemes are characterized by low dispersion as well as an ability to work on coarse grids. One such scheme is the upwind leapfrog scheme [8], which is time reversible and upwind biased. Therefore, it does not have any dissipation error and should be appropriate for long-range wave propagation. However, when the equation has a source term, the exact solution may no longer be time reversible, and it is not obvious that a leapfrog treatment is suitable for the unsteady linear problem. In fact, a time reversible algorithm is always unstable in these circumstances (see Section 2.3). Therefore, in the following sections we will report two different ways to discretise the source term and consequently to resolve the instability issues. This analysis may also be applicable in developing other low-dissipation schemes.

\section{ONE-DIMENSIONAL ADVECTION EQUATION}

For the one-dimensional advection problem, $u_{t}+a u_{x}=0$, the upwind leapfrog scheme can be written as

$$
u_{j}^{n+1}=u_{j-1}^{n-1}+(1-2 v)\left(u_{j}^{n}-u_{j-1}^{n}\right)
$$

* Correspondence to: Cheolwan Kim, 4433 Hillside Court, Ann Arbor, MI 48105, U.S.A.

Copyright (c) 2003 John Wiley \& Sons, Ltd.

Received 29 September 1998 Revised 13 February 2003 


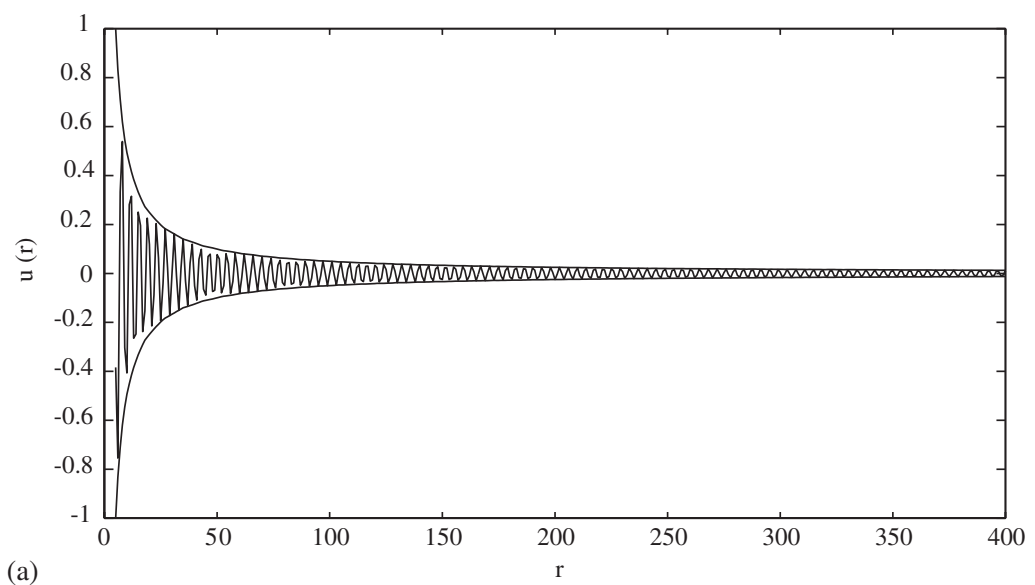

(a)

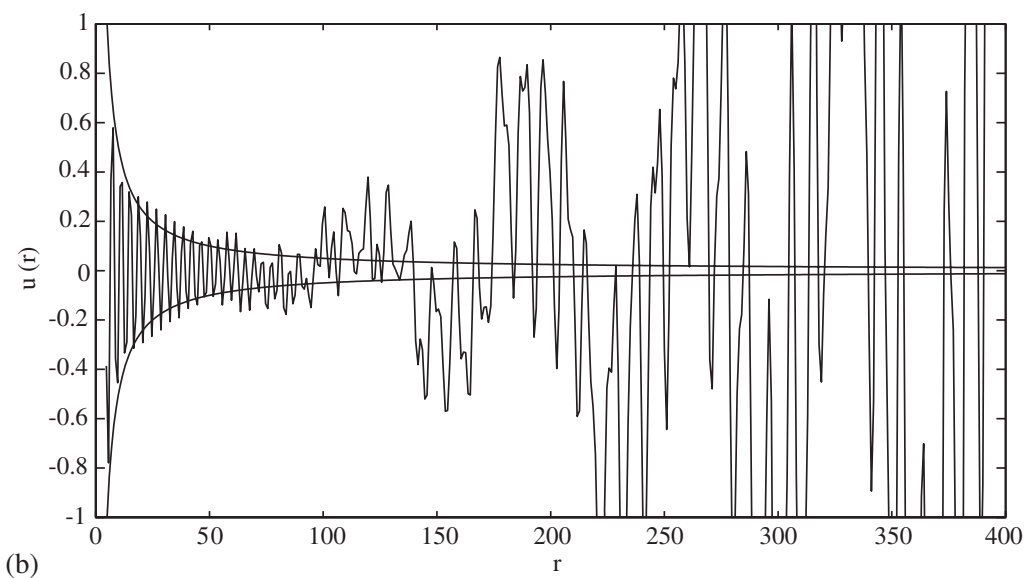

Figure 1. Spherical wave solutions: (a) Solution with exact damping. (b) Solution with simple average for source term.

where $v=a \Delta t / \Delta x$. It is time reversible and updates the solution without any dissipation error. When the advection equation has a source term where $s$ is assumed to be constant

$$
u_{t}+a u_{x}=s u
$$

a standard discretization is a simple average written as

$$
u_{j}^{n+1}=u_{j-1}^{n-1}+(1-2 v)\left(u_{j}^{n}-u_{j-1}^{n}\right)+s \Delta t\left(u_{j-1}^{n}+u_{j}^{n}\right)
$$

However, as shown in Figure 1(b), a numerical experiment indicates that a leapfrog treatment is unstable with a source term. This instability could be explained as follows.

Since there exist both physical and spurious solutions in any multilevel scheme, threelevel schemes such as Equations (1) or (3) also have physical and spurious solutions. While the physical solution provides a reasonable approximation to the continuous problem, the spurious solution is observed to oscillate in time and is triggered (a) by a poor starting 
procedure at the first time step, or (b) through aliasing caused by non-linearity, or (c) through the boundary procedures. The amplification factors of the physical and spurious modes are denoted, respectively, by $g_{\mathrm{p}}$ and $g_{\mathrm{s}}$ and the product of their moduli is given by

$$
\left|g_{\mathrm{p}}\right|\left|g_{\mathrm{s}}\right|=\left|\frac{C_{j-1}^{n-1}}{C_{j}^{n+1}}\right|=1
$$

where $C_{j}^{n}$ is the coefficient of $u_{j}^{n}$ in the difference equation (3). For negative $s$, the physical solution decays $\left(\mid g_{\mathrm{p}}<1\right)$ and the spurious solution grows $\left(\left|g_{\mathrm{s}}\right|>1\right)$. Even though the mechanism for introducing it may be very weak, it will eventually dominate the computation. A way to resolve the instability is suggested for the one-dimensional problems later in this section.

However, if $s$ is positive $(s>0),\left|g_{\mathrm{p}}\right|>1$ and $\left|g_{\mathrm{s}}\right|<1$. Therefore, discretization (3) produces a stable solution and it does not need any special treatment for the source term.

\subsection{Transformation of the governing equation}

A new variable $v$, defined by

$$
u=\mathrm{e}^{\alpha t+\beta x} v
$$

was introduced to transform the governing equation where $\alpha, \beta$ are constants to be determined. Substituting relation (5) in Equation (2) leads to

$$
v_{t}+a v_{x}+(\alpha+a \beta-s) v=0
$$

Letting $\alpha+a \beta-s=0$ eliminates the source term and yields a pure advection equation. To determine $\alpha$ and $\beta$, two simplest choices are made as below.

Case 1: $\alpha=s$ and $\beta=0 \rightarrow u=\mathrm{e}^{s t} v$.

Case 2: $\alpha=0$ and $\beta=s / a \rightarrow u=\mathrm{e}^{s x / a} v$.

The transformation with time and space variables $(\alpha \beta \neq 0)$ is not considered further since it makes the numerical scheme complicated and produces results intermediate between the two extreme cases.

2.1.1. Transformation with time variable (Case 1). Letting $\alpha=s, \beta=0$ in (6) leads to

$$
u=\mathrm{e}^{s t} v \Rightarrow v_{t}+a v_{x}=0
$$

Discretizing the above equation and translating back to $u$ yields a stable upwind leapfrog scheme for the advection equation with a source term.

$$
\mathrm{e}^{-s \Delta t} u_{j}^{n+1}=\mathrm{e}^{s \Delta t} u_{j-1}^{n-1}+(1-2 v)\left(u_{j}^{n}-u_{j-1}^{n}\right)
$$

The physical and spurious solutions of the above scheme have the same amplification factor

$$
\left|g_{\mathrm{p}}\right|=\left|g_{\mathrm{s}}\right|=\mathrm{e}^{s \Delta t} \approx 1+s \Delta t
$$

Therefore, the spurious solution grows or decays at the same rate as the physical solution and never dominates the computation domain. Linearization of $\mathrm{e}^{s \Delta t}(\approx 1+s \Delta t)$ gives a simpler 
form that shares the same property.

$$
u_{j}^{n+1}=u_{j-1}^{n-1}+(1-2 v)\left(u_{j}^{n}-u_{j-1}^{n}\right)+s \Delta t\left(u_{j}^{n+1}+u_{j-1}^{n-1}\right)
$$

Comparing the difference equations (3) and (10) reveals that the source term, $\left(u_{j-1}^{n}+u_{j}^{n}\right) / 2$ was replaced with $\left(u_{j-1}^{n-1}+u_{j}^{n+1}\right) / 2$. This transformation was also described by Roe [9].

2.1.2. Transformation with space variable (Case 2). With $\alpha=0, \beta=s / a$, the variable $v$, becomes

$$
u=\mathrm{e}^{s x / a} v \Rightarrow v_{t}+a v_{x}=0
$$

Discretizing the new advection equation through the upwind leapfrog scheme yields another stable scheme:

$$
\mathrm{e}^{-s \Delta x / 2 a} u_{j}^{n+1}=\mathrm{e}^{s \Delta x / 2 a} u_{j-1}^{n-1}+(1-2 v)\left(\mathrm{e}^{-s \Delta x / 2 a} u_{j}^{n}-\mathrm{e}^{s \Delta x / 2 a} u_{j-1}^{n}\right)
$$

Linearizing the exponential term yields

$$
\begin{aligned}
u_{j}^{n+1}= & u_{j-1}^{n-1}+(1-2 v)\left(u_{j}^{n}-u_{j-1}^{n}\right)+s \Delta t\left(u_{j}^{n}+u_{j-1}^{n}\right) \\
& +\frac{s \Delta x}{2 a}\left(u_{j}^{n+1}-u_{j}^{n}-u_{j-1}^{n}+u_{j-1}^{n-1}\right)
\end{aligned}
$$

which can be written

$$
\begin{aligned}
u_{j}^{n+1}= & u_{j-1}^{n-1}+(1-2 v)\left(u_{j}^{n}-u_{j-1}^{n}\right)+s \Delta t\left(1-\frac{1}{2 v}\right)\left(u_{j}^{n}+u_{j-1}^{n}\right) \\
& +\frac{s \Delta t}{2 v}\left(u_{j}^{n+1}+u_{j-1}^{n-1}\right)
\end{aligned}
$$

Equation (14) should coincide with the difference equation (10) if $v=\frac{1}{2}$.

\subsection{Stability analysis}

The amplification factors of a three-level scheme, $g_{\mathrm{p}}, g_{\mathrm{s}}$, are roots of a quadratic equation with complex coefficients, i.e.

$$
a g^{2}+2 b g+c=0
$$

whose roots are

$$
g=\frac{-b \pm \sqrt{b^{2}-a c}}{a}=\frac{-b_{r}-\mathrm{i} b_{i} \pm\left(d_{r}+\mathrm{i} d_{i}\right)}{a_{r}+\mathrm{i} a_{i}}
$$

where $d=\sqrt{b^{2}-a c}$. The moduli of the roots are equal if

$$
b=0 \quad \text { or } \quad d=0 \quad \text { or } \quad d^{2} / b^{2}<0
$$


Inserting the values of $b$ and $d$ for a particular scheme into relation (17) yields conditions that the amplification factor of the spurious solution is the same as that of the physical solution. For the transformation in time, (8), we can obtain the condition, $0<v<1$ with $d^{2} / b^{2}<0$. This is identical to the stability condition of the homogeneous scheme. For the transformation in space, (12), the moduli of roots are equal when $v=1 / 2(b=0)$ and the Fourier series analysis clarifies the following result:

$$
\begin{aligned}
& \left|g_{\mathrm{s}}\right| \leqslant\left|g_{\mathrm{p}}\right|<1, \quad 0<v \leqslant \frac{1}{2} \\
& \left|g_{\mathrm{p}}\right| \leqslant\left|g_{\mathrm{s}}\right|<1, \quad \frac{1}{2} \leqslant v<1
\end{aligned}
$$

where $s$ is negative. If we adopt the stability condition that the magnitude of the amplification factor for the spurious solution should be no greater than that of the physical solution, the difference equation (12) is stable only when $0<v \leqslant 1 / 2$. With the less strict definition that the spurious solution should not grow, i.e. $\left|g_{\mathrm{s}}\right|<1$, the stability region extends to $0<v<1$. Such a definition could be motivated by considering that the solution becomes meaningless when the waves have decayed to the level of whatever effect is responsible for introducing the spurious mode. This was proved by A. Hindmarsh and described in the appendix.

For positive $s$,

$$
\begin{aligned}
& \left|g_{\mathrm{s}}\right| \geqslant\left|g_{\mathrm{p}}\right|>1, \quad 0<v \leqslant \frac{1}{2} \\
& \left|g_{\mathrm{p}}\right| \geqslant\left|g_{\mathrm{s}}\right|>1, \quad \frac{1}{2} \leqslant v<1
\end{aligned}
$$

In the case $s>0$, a scheme should be stable if both modes have $|g|>1$, i.e. both modes decay when integrated backwords in time. Therefore, the discretized equation (12) is stable for either sign of $s$ when $0<v<1$.

\subsection{Simulation of spherical wave equation}

Since the spherical wave equation, $u_{t}+u_{r}=-u / r$, has a source term proportional to its solution and the exact solution, it is a good example to apply and examine the stabilizing techniques explained previously. Figure 1 shows the exact solution and an unstable numerical result obtained by implementing scheme (3). The computational domain $(5<r<400)$ is excited at the left boundary by a sinusoidal oscillation, $u_{r=5}^{n}=\sin (2 \pi v n / N)$. The source term coefficient, $1 / r$, is taken as the value of the stencil centre, $1 / r_{j-1 / 2}$. The instability sets in after a few hundred time steps. The error, which is very small initially, is excited by the spurious solution, grows very rapidly and overwhelms the physical solution.

To resolve the instability shown in Figure 1(b), stabilizing techniques are applied. Since the source term coefficient is, however, variable, a heuristic approximation that the source term coefficient is locally constant around $r_{j-1 / 2}$, should satisfy the assumption that the coefficient, $s$ is constant. Therefore, the new governing equation should be

$$
u_{t}+u_{r}=-\frac{1}{r_{j-1 / 2}} u
$$



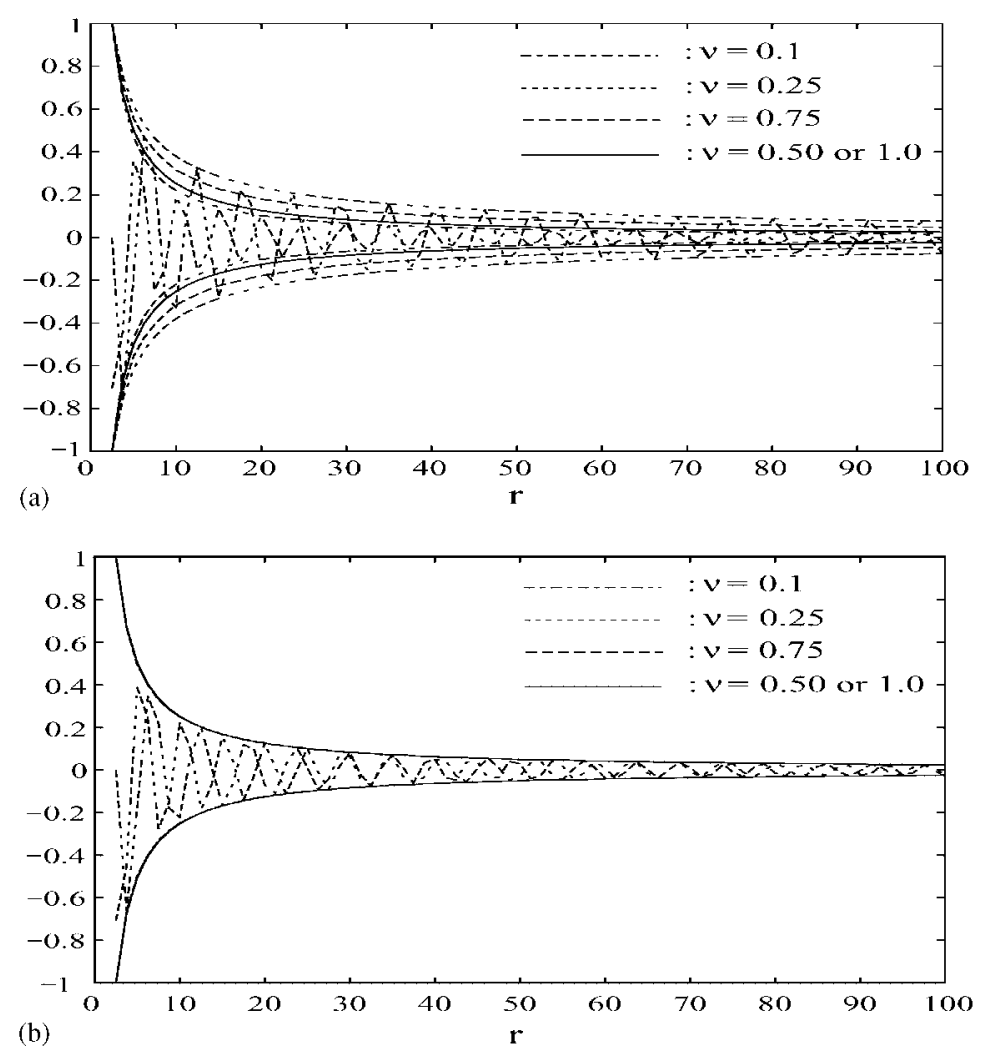

Figure 2. Envelopes of spherical wave solutions with second-order schemes for various Courant numbers, grid points per wavelength $=4$ : (a) Transformation with time. (b) Tansformation with space.

and valid in the range $r_{j-1}<r<r_{j}$ or $r_{j-2}<r<r_{j+1}$. Figures 2 and 3 compare the envelopes of the numerical solutions of two stable schemes (10) and (12) for various Courant numbers and grid points per wavelength, respectively. The parameter $N$ corresponds to the number of grid points per wavelength. Figure 2(a) shows the envelopes of the difference equation (10) for various Courant numbers. Although this scheme produces stable solutions, the envelope is dependent on the Courant numbers and agrees with the analytic solution only when $v=1 / 2$ or 1 . When $v<1 / 2$, the envelope is larger and less than the exact one when $1 / 2<v<1$. Figure 2(b) presents the envelopes of scheme (12). They match well with the analytic one regardless of the Courant numbers. Figure 3 shows the computed envelopes for various grid points per wavelength. Again the envelopes of the scheme from the transformation with time variable $t$, are dependent on the number of grid point per wavelength. The above comparison confirms that the transformation with the space variable predicts the envelope very well for all values of $v$ and even with $N$ as small as 4 .

The amplification factors of Case 2 is $\mathrm{e}^{s \Delta x / a}$ per wave propagation over $\Delta x$ and independent of the Courant number. Therefore, it predicts the magnitude of the solution very precisely. However, the amplification factor of Case 1 is $\mathrm{e}^{s \Delta t}$ per step and it is a little different from 


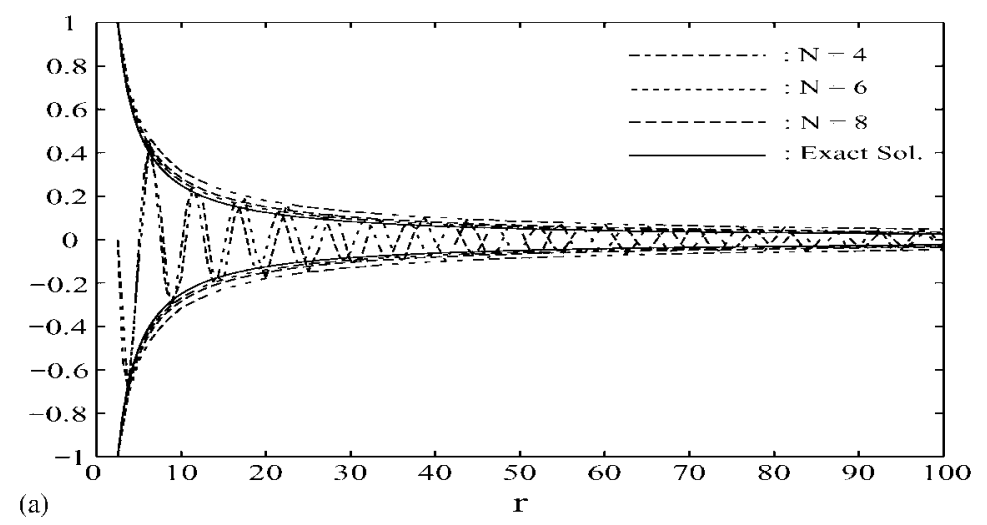

(a)

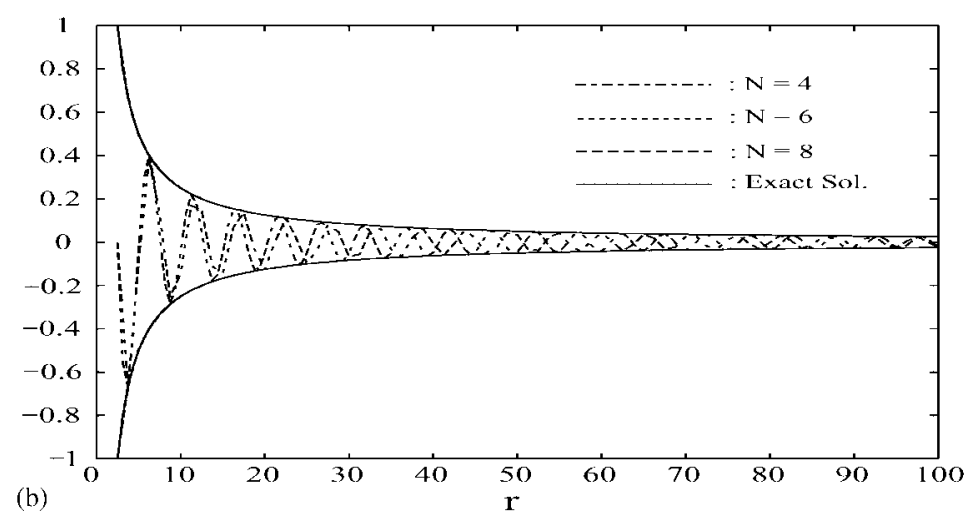

Figure 3. Envelopes of spherical wave solutions with second-order schemes for various grids per wavelength, Courant number $=1 / 4$ : (a) Transformation with time. (b) Transformation with space.

that of Case 2 after wave traveling $\Delta x$. The numerical solution travels $a_{\mathrm{N}} \Delta t$ per time step and it requires $\Delta x / a_{\mathrm{N}} \Delta t$ steps to travel $\Delta x$, where $a_{\mathrm{N}}$ is the numerical wave speed of the physical solution and defined by the relation $g_{\mathrm{p}} /\left|g_{\mathrm{p}}\right|=\exp \left(-\mathrm{i} \theta a_{\mathrm{N}} \Delta t / \Delta x\right)$. Therefore, the resultant amplification factor of Case 1 is $\mathrm{e}^{s \Delta x / a_{\mathrm{N}}}$. The numerical speed of the upwind leapfrog scheme, $a_{\mathrm{N}}$ is faster or slower than the analytic speed, $a$ when $0<v<1 / 2$ and $1 / 2<v<1$, respectively. Therefore, for negative $s$,

$$
\begin{array}{ll}
\mathrm{e}^{s \Delta x / a_{\mathrm{N}}}>\mathrm{e}^{s \Delta x / a}, & 0<v<1 / 2 \\
\mathrm{e}^{s \Delta x / a_{\mathrm{N}}}<\mathrm{e}^{s \Delta x / a}, & 1 / 2<v<1
\end{array}
$$

The above result is confirmed in Figure 2. The envelopes are over- and under-predicted by Equation (8) for $v<1 / 2$ and $v>1 / 2$, respectively, whereas Equation (12) predicts the envelopes precisely at all Courant numbers. Of course, the predictions from Case 1 improve as $N$ increases, since $a_{\mathrm{N}}$ approaches $a$. 


\section{TWO-DIMENSIONAL ACOUSTICS}

In two-dimensional acoustics, the governing equations have three variables, pressure $p$ and two velocities $u, v$. They are written in Cartesian co-ordinates as

$$
\begin{aligned}
\frac{\partial p}{\partial t}+\rho_{0} a_{0}^{2}\left(\frac{\partial u}{\partial x}+\frac{\partial v}{\partial y}\right) & =0 \\
\frac{\partial u}{\partial t}+\frac{1}{\rho_{0}} \frac{\partial p}{\partial x} & =0 \\
\frac{\partial v}{\partial t}+\frac{1}{\rho_{0}} \frac{\partial p}{\partial y} & =0
\end{aligned}
$$

where $\rho_{0}$ and $a_{0}$ are steady values of density and speed of sound, and are set to one. In order to apply the upwind leapfrog method to acoustics, the above Equations (22) are rearranged in characteristic form:

$$
\begin{gathered}
\frac{\partial(p \pm u)}{\partial t} \pm \frac{\partial(p \pm u)}{\partial x}=-\frac{\partial v}{\partial y} \\
\frac{\partial(q \pm v)}{\partial t} \pm \frac{\partial(q \pm v)}{\partial y}=-\frac{\partial u}{\partial x}
\end{gathered}
$$

Here $q$ is a second pressure treated as an independent variable, introduced to equalize the number of equations and unknowns when the discretization is applied to the staggered grid shown in Figure 4.

The difference equation can be produced by discretizing the equation for $p+u$ wave on the stencil of Figure 4.

For $(p+u)$ wave,

$$
\begin{aligned}
(p+u)_{j+1 / 2, k}^{n+1}= & (p+u)_{j-1 / 2, k}^{n-1}+\left(1-2 v_{x}\right)\left[(p+u)_{j+1 / 2, k}^{n}-(p+u)_{j-1 / 2, k}^{n}\right] \\
& -2 v_{y}\left(v_{j, k+1 / 2}^{n}-v_{j, k-1 / 2}^{n}\right)
\end{aligned}
$$

where $v_{x}=\Delta t / \Delta x$ and $v_{y}=\Delta t / \Delta y$. Other equations can be discretized in the same manner.

\subsection{Stable discretization of the acoustic equations in polar co-ordinates}

In polar co-ordinates $(r, \theta, t)$ with velocity components $u$ and $v$, the acoustic equations in characteristic form are

$$
\begin{gathered}
\frac{\partial(p \pm u)}{\partial t} \pm \frac{\partial(p \pm u)}{\partial r}+\frac{1}{r} \frac{\partial v}{\partial \theta}=-\frac{u}{r} \\
\frac{\partial(q \pm v)}{\partial t} \pm \frac{1}{r} \frac{\partial(q \pm v)}{\partial \theta}+\frac{\partial u}{\partial r}=-\frac{u}{r}
\end{gathered}
$$





Figure 4. Stencils of staggered grid for second-order upwind leapfrog methods.

$p$ and $q$ are the pressures updated with $u$ and $v$, respectively, and these equations have source terms on the right-hand side. Previously, two techniques were suggested to stabilize the source term of the advection equations. The techniques are to transform the dependent variables to include either the temporal or space variable and so to eliminate the source term. In the acoustic problem, transformation with the temporal variable turned out to be unsuccessful. However, the governing equations transformed with the space variable can be successfully discretized and generate stable solutions for long-range acoustic problems. To transform Equations (26), (27) with a space variable, they are split and written as the system of equations:

$$
\begin{gathered}
\frac{\partial \mathbf{W}_{r}}{\partial t}+\Lambda_{r} \frac{\partial \mathbf{W}_{r}}{\partial r}+\frac{1}{r} \frac{\partial v}{\partial \theta} \mathbf{E}=\mathbf{S} \mathbf{W}_{r} \\
\frac{\partial \mathbf{W}_{\theta}}{\partial t}+\boldsymbol{\Lambda}_{\theta} \frac{\partial \mathbf{W}_{\theta}}{\partial \theta}+\frac{\partial u}{\partial r} \mathbf{E}=\mathbf{S} \mathbf{W}_{r}
\end{gathered}
$$

where

$$
\begin{array}{ll}
\mathbf{W}_{r}=\left[\begin{array}{l}
W_{r}^{+} \\
W_{r}^{-}
\end{array}\right]=\left[\begin{array}{l}
p+u \\
p-u
\end{array}\right], \quad \boldsymbol{\Lambda}_{r}=\left[\begin{array}{cc}
1 & 0 \\
0 & -1
\end{array}\right], \quad \mathbf{S}=\frac{1}{2 r}\left[\begin{array}{ll}
-1 & 1 \\
-1 & 1
\end{array}\right] \\
\mathbf{W}_{\theta}=\left[\begin{array}{l}
W_{\theta}^{+} \\
W_{\theta}^{-}
\end{array}\right]=\left[\begin{array}{l}
q+v \\
q-v
\end{array}\right], \quad \boldsymbol{\Lambda}_{\theta}=\frac{1}{r}\left[\begin{array}{cc}
1 & 0 \\
0 & -1
\end{array}\right], \quad \mathbf{E}=\left[\begin{array}{l}
1 \\
1
\end{array}\right]
\end{array}
$$

Equation (28) has a source term which is proportional to the variables, $\mathbf{W}_{r}$ and it requires the stabilizing technique. However Equation (29) has no source term proportional to $\mathbf{W}_{\theta}$ and can be averaged simply. In fact, a discretization that is symmetric about the centroid of the stencil shown in Figure 5(b) yields stable schemes for the waves propagating in the circumferential direction. The second-order accurate difference equations based on Figure 5(b) are

$$
\begin{aligned}
& \left.W_{\theta}^{+}\right|_{a}=\left.W_{\theta}^{+}\right|_{b}+\left(1-2 v_{\theta}\right)\left(\left.W_{\theta}^{+}\right|_{2}-\left.W_{\theta}^{+}\right|_{3}\right)-2 v_{r}\left(u_{5}-u_{7}\right)-\Delta t\left(u_{5}+u_{7}\right) / r_{c} \\
& \left.W_{\theta}^{-}\right|_{a}=\left.W_{\theta}^{-}\right|_{c}+\left(1-2 v_{\theta}\right)\left(\left.W_{\theta}^{-}\right|_{2}-\left.W_{\theta}^{-}\right|_{1}\right)-2 v_{r}\left(u_{4}-u_{6}\right)-\Delta t\left(u_{4}+u_{6}\right) / r_{c}
\end{aligned}
$$

where $v_{\theta}=\Delta t / r_{c} \Delta \theta$ and $v_{r}=\Delta t / \Delta r$. 



(a)

(b)

Figure 5. Second-order stencils for acoustics in polar co-ordinate: (a) For wave moving in $r$-direction. (b) For wave moving in $\theta$-direction.

Before applying the stabilizing technique to Equation (28), another heuristic approximation that $S$ is locally constant around $r_{2}$, is made to satisfy the assumption of the technique. A new vector, $V$ defined by

$$
\mathbf{W}_{r}=\mathrm{e}^{\mathbf{D} r} \mathbf{V}
$$

leads to a system of equations containing no source term.

$$
\mathrm{e}^{\mathbf{D} \cdot r} \frac{\partial \mathbf{V}}{\partial t}+\boldsymbol{\Lambda}_{\mathbf{r}} \mathrm{e}^{\mathbf{D} \cdot r} \frac{\partial \mathbf{V}}{\partial r}+\frac{1}{r} \frac{\partial v}{\partial \theta} \mathbf{E}=0
$$

where

$$
\mathbf{D}=\boldsymbol{\Lambda}_{r}^{-1} \mathbf{S}=\frac{1}{2 r_{2}}\left[\begin{array}{cc}
-1 & 1 \\
1 & -1
\end{array}\right]
$$

Translating back to the original variable, $W_{r}$ presents

$$
\mathrm{e}^{\mathbf{D} r} \frac{\partial}{\partial t}\left(\mathbf{e}^{-\mathbf{D} r} \mathbf{W}_{r}\right)+\boldsymbol{\Lambda}_{r} \mathrm{e}^{\mathbf{D} r} \frac{\partial}{\partial r}\left(\mathbf{e}^{-\mathbf{D} r} \mathbf{W}_{r}\right)+\frac{1}{r} \frac{\partial v}{\partial \theta} \mathbf{E}=0
$$

The implementation of Equation (31) is confined to the local area around $r_{2}$ and it can be replaced with

$$
\mathrm{e}^{\mathbf{D} r_{2}} \frac{\partial}{\partial t}\left(\mathbf{e}^{-\mathbf{D} r} \mathbf{W}_{r}\right)+\boldsymbol{\Lambda}_{r} \mathrm{e}^{\mathbf{D} r_{2}} \frac{\partial}{\partial r}\left(\mathbf{e}^{-\mathbf{D} r} \mathbf{W}_{r}\right)+\frac{1}{r_{2}} \frac{\partial v}{\partial \theta} \mathbf{E}=0
$$

Furthermore, it can be simplified as

$$
\frac{\partial\left(\mathrm{e}^{-\mathbf{D}\left(r-r_{2}\right)} \mathbf{W}_{r}\right)}{\partial t}+\boldsymbol{\Lambda}_{r} \frac{\partial\left(\mathrm{e}^{-\mathbf{D}\left(r-r_{2}\right)} \mathbf{W}_{r}\right)}{\partial r}+\frac{1}{r_{2}} \frac{\partial v}{\partial \theta} \mathbf{E}=0
$$


This is the system of characteristic equations for the waves moving along the radial direction. A linear approximation of $\mathrm{e}^{\mathbf{D}\left(r-r_{2}\right)}\left(\approx 1+\mathbf{D}\left(r-r_{2}\right)\right)$ simplifies the equations:

$$
\begin{aligned}
& \frac{\partial(p+u+\bar{r} u)}{\partial t}+\frac{\partial(p+u+\bar{r} u)}{\partial r}+\frac{1}{r_{2}} \frac{\partial v}{\partial \theta}=0 \\
& \frac{\partial(p-u-\bar{r} u)}{\partial t}-\frac{\partial(p-u-\bar{r} u)}{\partial r}+\frac{1}{r_{2}} \frac{\partial v}{\partial \theta}=0
\end{aligned}
$$

where $\bar{r}=\left(r-r_{2}\right) / r_{2}$. Discretizing these equations in Figure 5(a) yields stable upwind leapfrog schemes for waves propagating in the radial direction.

$$
\begin{aligned}
& \frac{(p+u)_{a}-(p+u)_{2}+(p+u)_{3}-(p+u)_{b}}{2 \Delta t}+\frac{\Delta r\left(u_{a}-u_{2}-u_{3}+u_{b}\right)}{4 \Delta t r_{2}} \\
& +\frac{(p+u)_{2}-(p+u)_{3}}{\Delta r}+\frac{1}{r_{2}} \frac{v_{5}-v_{7}}{\Delta \theta}+\frac{1}{2 r_{2}}\left(u_{2}+u_{3}\right)=0 \\
& \frac{(p-u)_{a}-(p-u)_{2}+(p-u)_{1}-(p-u)_{c}}{2 \Delta t}-\frac{\Delta r\left(u_{a}-u_{2}-u_{1}+u_{c}\right)}{4 \Delta t r_{2}} \\
& -\frac{(p-u)_{1}-(p-u)_{2}}{\Delta r}+\frac{1}{r_{2}} \frac{v_{4}-v_{6}}{\Delta \theta}+\frac{1}{2 r_{2}}\left(u_{1}+u_{2}\right)=0
\end{aligned}
$$

This stabilizing technique can be applied to fourth-order schemes in a similar way.

\subsection{Numerical experiment}

To test the technique described above, an initial-value problem was simulated on a polar grid to prove the robustness of the scheme in an extreme case. The initial disturbance is

$$
\begin{aligned}
& p(x, y)=\exp \left(-\frac{\ln ^{2}}{0.2^{2}}\left[(x-4)^{2}+y^{2}\right]\right) \\
& u(x, y)=v(x, y)=0
\end{aligned}
$$

The computational domain (Figure $6(\mathrm{a})$ ) is $r \leqslant 6.0,0 \leqslant \theta \leqslant 2 \pi$ and the mesh size is $60 \times 60$ mesh; which is rather coarse. A polar grid is, of course, quite inappropriate for this problem, and most of the methods that we examined, including transformation (7) became rapidly unstable. Since the time step is restricted by the smallest cells, the time step is $\pi \Delta r / 60$. The whole computational domain is inside the circle whose radius is 6.0 , but pressure contours for the region $(-3 \leqslant x \leqslant 3,-3 \leqslant y \leqslant 3)$ are presented to magnify the area around the origin. Since the centre of the pulse is at $(4,0)$, the pressure peak moving in the negative direction is just shown in the pictures. When $t=2$, the pressure peak reaches the position, $x=2$ and the wave front is not circular because the grid is very coarse around the origin (Figure 6(b)). Then the peak passes the origin without any instability and reaches the point $(-2,0)$ at $t=6$, Figure $6(\mathrm{~d})$. When $t=8$, the main pressure peak disappears from the domain, leaving only a slight disturbance in the domain. 




(a)

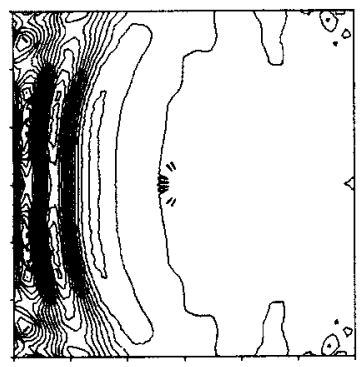

(d)



(b)

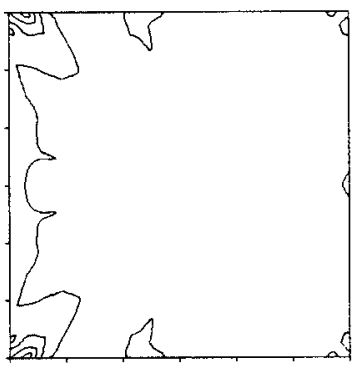

(e)

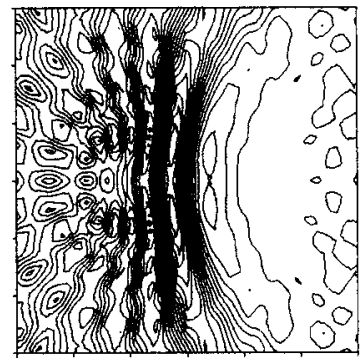

(c)

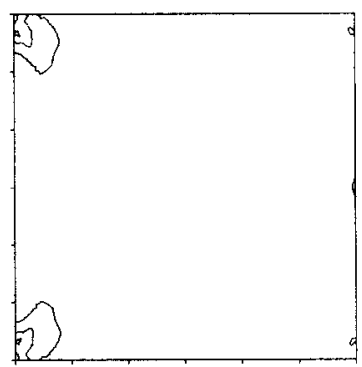

(f)

Figure 6. Test of stabilizing technique with cylindrical co-ordinates including co-ordinate origin, $0 \leqslant r \leqslant 6,0 \leqslant \theta \leqslant 2 \pi$, grid size: $60 \times 60$ : (a) $t=0$. (b) $t=2$. (c) $t=4$. (d) $t=6$. (e) $t=8$. (f) $t=10$.

\section{CONCLUSION}

Two stabilizing techniques to discretize the source terms of the one-dimensional advection equation and two-dimensional acoustic equations have been compared. To eliminate the source terms, each method transforms the governing equation. The first method transforms the governing equation through the time variable. Its damping rate for propagating waves is dependent on the time step. It was found that the transformation using the time variable did not work for the two-dimensional acoustic wave system. The transformation using the space variable, however, predicts the amplitude of the wave envelopes regardless of the Courant number and has been applied to the two-dimensional system of equations in polar co-ordinates successfully.

\section{APPENDIX: STABILITY ANALYSIS BY A. C. HINDMARSH ${ }^{\dagger}$}

The following provides stability results for Equation (12).

\section{Theorem}

The two amplification factors $g$ for the discretized scheme in Equation (12), from Equations (15)-(16), satisfy the following: (a) For $0<v<1, \max |g|<1$ for $s<0$, and $\min |g|>1$ for

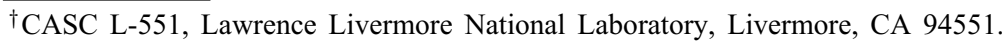


$s>0$. (b) For $v=0$ or $1, \max |g|=1$ for $s<0$, and $\min |g|=1$ for $s>0$. (c) For any $v<0$ or $v>1, \min |g|<1<\max |g|$ for a non-zero $s$ of either sign.

Proof

Define $r=\exp (s \Delta x / 2 a)$ and $\mu=1-2 v$. Then the difference equation (12) is

$$
r^{-1} u_{j}^{n+1}=r u_{j-1}^{n-1}+\mu\left(r^{-1} u_{j}^{n}-r u_{j-1}^{n}\right)
$$

Inserting a Fourier mode $u_{j}^{n}=g^{n} \mathrm{e}^{\mathrm{i} j \phi}$, we get the quadratic $a g^{2}+2 b g+c=0$ with coefficients $a=r^{-1}, 2 b=\mu\left(r \mathrm{e}^{-\mathrm{i} \phi}-r^{-1}\right), c=-r \mathrm{e}^{-\mathrm{i} \phi}$. Defining $\theta=-\phi / 2$ and $z=r \mathrm{e}^{\mathrm{i} \theta}$, the quadratic is

$$
0=(a / c) g^{2}+(2 b / c) g+1=-z^{-2} g^{2}+\mu\left(z^{-2}-1\right) g+1
$$

It helps now to change unknowns to $G=z^{-1} g$. Then we have

$$
-G^{2}+\mu\left(z^{-1}-z\right) G+1=0 \quad \text { or } \quad G-G^{-1}=-\mu\left(z-z^{-1}\right)
$$

Now make use of the well-known conformal map $w=f(z)=\left(z-z^{-1}\right) / 2$, which maps the unit disk $|z|<1$ conformally onto the exterior of the slit from $-i$ to $i$ in the $w$ plane. For $r<1$, it maps the circle $C(r)=\{|z|=r\}$ onto the ellipse $E(r)$ given by $w=\frac{1}{2}\left(r-r^{-1}\right) \cos \theta+$ $\frac{1}{2}\left(r+r^{-1}\right) i \sin \theta$, with vertical axis $r+r^{-1}$ and horizontal axis $\left|r^{-1}-r\right|$. The exterior of the unit disk in the $z$ plane is also mapped by $f$ onto the same slit plane, with $C\left(r^{-1}\right)$ being mapped onto $E\left(r^{-1}\right)$, which is identical to $E(r)$. For $r$ near $1, E(r)$ is a tall and narrow ellipse near the slit, which is $E(1)$. For $r$ near either 0 or $\infty, E(r)$ is large and nearly circular. For a given $z=r \mathrm{e}^{\mathrm{i} \theta}$, setting $w=f(z)$ and $\hat{w}=-\mu w$, the equation for $G$ becomes

$$
f(G)=\left(G-G^{-1}\right) / 2=-\mu\left(z-z^{-1}\right) / 2=-\mu f(z)=-\mu w=\hat{w}
$$

So finding the roots $G$ is equivalent to finding the pre-images of $\hat{w}=-\mu w$ under the mapping $f$.

First consider the case $s<0,1 / 2<v<1$. Then $r<1$ and $-1<\mu<0$. For the moment, assume that $\mathfrak{R}(w) \neq 0$. Since $w$ lies on $E(r)$ and $0<-\mu<1$, we see that $\hat{w}$ lies on an ellipse $E(\hat{r})$ in the same family of ellipses, lying in between $E(r)$ and the slit $E(1)$. Thus, it is an $E(\hat{r})$ with $r<\hat{r}<1$. The pre-images of $\hat{w}$ are on the circles $C(\hat{r})$ and $C\left(\hat{r}^{-1}\right)$. Hence, the moduli of the two roots are $|G|=\hat{r}, \hat{r}^{-1}$. The larger root satisfies $\max |G|=\hat{r}^{-1}<r^{-1}$, and so $\max |g|=|z| \max |G|=r \hat{r}^{-1}<1$. If $\mathfrak{R}(w)=0$ (i.e. $w$ is on the imaginary axis), we find that $z$ must be $z= \pm \mathrm{i} r$ and $w= \pm \mathrm{i}\left(r+r^{-1}\right) / 2$, and $\hat{w}=-\mu w$ is also on the imaginary axis. If $|\hat{w}|=|\mu|\left(r+r^{-1}\right) / 2>1$, then $\hat{w}$ again lies on an ellipse $E(\hat{r})$ with $r<\hat{r}<1$, and the result follows as before. If $|\hat{w}| \leqslant 1$, then $\hat{w}$ lies on the slit $E(1)$, and its pre-images $G$ are two points on the unit circle $C(1)$ (or a double root $G= \pm \mathrm{i}$ in the cases $\hat{w}= \pm \mathrm{i}$ ). Then $|G|=1$ and $|g|=r<1$ for both roots.

Now consider the case $s<0,0<v<1 / 2$. Then $r<1$ and $0<\mu<1$. If $\Re(w) \neq 0$, then again $\hat{w}=-\mu w$ lies on an $E(\hat{r})$, with $r<\hat{r}<1$, but on the opposite side of the origin from $w$. Again $|G|=\hat{r}, \hat{r}^{-1}$ for the two roots, and $\max |g|=r \max |G|=r \hat{r}^{-1}<1$. If $\mathfrak{R}(w)=0$, then again $\hat{w}=-\mu w$ is also on the imaginary axis, and, depending on the value of $\mu$, either $|G|=\hat{r}, \hat{r}^{-1}$ and $\max |g|=r \hat{r}^{-1}<1$, or $|G|=1$ and $|g|=r<1$ for both roots.

The results for $s>0(r>1)$ can be proven in the same manner. Given $0<|\mu|<1$ and $z=r \mathrm{e}^{\mathrm{i} \theta}, w=f(z)$ lies on $E(r)$, and $\hat{w}=-\mu w$ lies on an $E(\hat{r})$ with $r>\hat{r}>1$ and $|g|=r \hat{r}, r \hat{r}^{-1}$, 
except for special cases where $\hat{w}$ lies on the slit and $|g|=r$. In either case, $\min |g|>1$. One can also get these results from those for $s<0$ by use of symmetry: Changing $r$ to $r^{-1}$ and $\theta$ to $-\theta$ in the quadratic for $g$ changes $z$ to $z^{-1}$ and $g$ to $g^{-1}$.

Three special cases are easily verified directly: For $v=1 / 2(\mu=0)$, the roots are clearly $G= \pm 1$, or $g= \pm z$, and so $|g|=r$. For $v=0(\mu=1)$, the roots are $G=-z, z^{-1}$, or $g=-z^{2}, 1$. For $v=1(\mu=-1)$, the roots are $G=z,-z^{-1}$, or $g=z^{2},-1$. So for $v=0$ or 1 , the values of $|g|$ are 1 and $r^{2}$.

Finally, for $v<0$ or $v>1$, i.e. $|\mu|>1, \hat{w}$ lies on an ellipse $E(\hat{r})$ that is outside of $E(r)$. Thus we can take $\hat{r}<r<1$ if $s<0$, and $\hat{r}>r>1$ if $s>0$. In either case, $\max |g|=\max \left(r \hat{r}, r \hat{r}^{-1}\right)>1$, and $\min |g|=\min \left(r \hat{r}, r \hat{r}^{-1}\right)<1$.

\section{ACKNOWLEDGEMENTS}

I would like to express my deep appreciation to Professor P. L. Roe for his insight and thoughtful discussion on this research. I also appreciate Dr Alan Hindmarsh for his proof in the appendix.

\section{REFERENCES}

1. Davis S. Low-dispersion finite difference methods for acoustic waves in a pipe. Journal of the Acoustical Society of America 1991; 90(5):2775-2781.

2. Haras Z, Ta'asan S. Finite-difference schemes for long-time integration. Journal of Computational Physics 1994; 114:265-279.

3. Hu FQ, Hussaini MY, Manthey J. Low-dissipation and dispersion Runge-Kutta schemes for computational acoustics. Journal of Computational Physics 1996; 124:177-191.

4. Kopriva DA. Spectral solution of the time dependent Euler gasdynamics equations. AIAA Paper DGLR/AIAA92-02-036 (DGLR/AIAA 14th Aeroacoustics Conference), 1992.

5. Lele SK. Compact finite difference schemes with spectral-like resolution. Journal of Computational Physics 1991; 103:16-42.

6. Tam CKW, Webb JC. Dispersion-relation-preserving finite difference schemes for computational acoustics. Journal of Computational Physics 1992; 107:262-281.

7. Zingg DW, Lomax H, Jurgens HM. High accuracy finite-difference scheme for linear wave propagation. SIAM Journal on Scientific Computing 1996; 17:328-346.

8. Thomas JP, Roe PL. Development of non-dissipative numerical schemes for computational aeroacoustics. AIAA Paper 93-3382 (AIAA 11th Computational Fluid Dynamics Conference), 1993.

9. Roe PL. Linear bicharacteristic schemes without dissipation. SIAM Journal of Scientific Computing 19(5).

10. Tam CKW, Hardin JC. (eds). Second Computational Aeroacoustics (CAA) Workshop on Benchmark Problems, NASA Conference Paper 3352, June 1997. 